\title{
Study of Platelet Indices in Patients with Metabolic Syndrome
}

\author{
Uma Maheswara Reddy ${ }^{1}$ Aditya Khandekar ${ }^{1}$ Sourya Acharya ${ }^{1}$ Samarth Shukla ${ }^{2}$ Neema Acharya ${ }^{3}$
}

${ }^{1}$ Department of Internal Medicine, Jawaharlal Nehru Medical College, Wardha, Maharashtra, India

2Department of Pathology, Jawaharlal Nehru Medical College, Wardha, Maharashtra, India

${ }^{3}$ Department of Obstetrics and Gynecology, Jawaharlal Nehru Medical College, Wardha, Maharashtra, India

\author{
Address for correspondence Aditya Khandekar, Jawaharlal Nehru \\ Medical College, Wardha 442001, Maharashtra, India \\ (e-mail: adityajnmc@gmail.com).
}

Int J Recent Surg Med Sci 2019;5:10-14

\begin{abstract}
Keywords

- metabolic syndrome

- atherosclerosis

- platelet indices

- triglycerides

Introduction Metabolic syndrome (MetS) is a combination of abdominal obesity, atherogenic dyslipidemia, elevated blood pressure, and elevated plasma glucose. MetS patients have higher chances of developing insulin resistance, visceral adiposity, atherogenic dyslipidemia, and thus coronary artery disease and stroke. Alteration of platelet indices in diabetes mellitus, atherosclerosis, and other proinflammatory states has been described in multiple studies. Thus, this study was carried out to assess platelet indices in MetS.

Objectives This study was carried out to assess platelet indices in MetS.

Methods A cross-sectional study was carried out at Acharya Vinoba Bhave Rural Hospital, a tertiary care center over a period of 2 months from June 1, 2018 to July 31, 2018. Fifty patients diagnosed as having MetS, and 50 healthy controls were chosen. Estimation of anthropometric parameters including waist circumference; measurement of blood pressure; biochemical parameters including lipid profile; and platelet indices including plateletcrit, mean platelet volume (MPV), and platelet distribution width (PDW) were carried out.

Statistical Analysis Statistical analyses were carried out using inferential statistics, including chi-square test and Student's unpaired $t$-test, and software SPSS version 22.0 (IBM Corporation, Armonk, New York, United States) with GraphPad Prism version 6.0 (Informer Technologies, Inc. Los Angeles, California, United States) was used, with $p<0.05$ being considered as significant.

Results A statistically significant, positive correlation was found between the waist circumference, systolic blood pressure, serum triglyceride levels, and plateletcrit, with the MetS status of patients $(p<0.05)$.

Conclusion This study revealed that MetS is a proinflammatory and prothrombotic state, characterized by alteration of platelet indices. Plateletcrit was shown to be a statistically significant biomarker along with other parameters such as waist circumference, systolic blood pressure, and serum triglyceride levels. Early detection and follow-up of patients using these markers can lead to an overall decline in morbidity and mortality owing to MetS.
\end{abstract}

\section{Introduction}

Metabolic syndrome (MetS) is a combination of abdominal obesity, atherogenic dyslipidemia, elevated blood pressure
(BP), and elevated plasma glucose. ${ }^{1}$ Prevalence of the MetS in India varies according to extent of urbanization, with some studies reporting that up to one-third of the urban population

\section{received}

November 2, 2018

accepted

February 19, 2019
License terms

(우 (1) $\Theta \circledast$ 
in large cities in India have MetS. ${ }^{2,3}$ Studies from South India have shown the prevalence of classic risk factors for MetS to be $31.4 \%$ for abdominal obesity, $45.6 \%$ for hypertriglyceridemia, $65.5 \%$ for low high-density lipoprotein (HDL), 55.4\% for hypertension, and $26.7 \%$ having raised fasting plasma glucose. ${ }^{4,5}$ Some of the studies from eastern India have estimated a prevalence of MetS to be $31.4 \%$ in their study population, with female population having a higher prevalence than male population. ${ }^{6}$ The rural prevalence of MetS is found to be reasonably low when compared with the urban prevalence. ${ }^{7}$

Recent studies on platelet volume indices in the spectrum of ischemic heart diseases show that all platelet indices-plateletcrit, mean platelet volume (MPV) and platelet distribution width (PDW)-were significantly raised in patients who had suffered from acute myocardial ischemia. A key component of vaso-occlusion is believed to be increased platelet activation and reactivity, and thus an increased platelet volume resulting in an elevated PDW. Also, higher concentrations of platelet microparticles have been detected in proinflammatory disorders such as sickle cell anemia. Studies on diabetes mellitus (DM), which too is considered to be a prothrombotic state with enhanced platelet reactivity, found a statistical rise in MPV and PDW, yet very few studies have been carried out regarding platelet indices for MetS patients.

MetS patients have been found to be at a greater risk for developing insulin resistance, visceral adiposity, atherogenic dyslipidemia, and endothelial dysfunction. Patients with insulin resistance tend to develop hypertriglyceridemia and are prone for developing atheromas in vascular lumen, leading to an increased incidence of coronary artery disease and stroke. ${ }^{8}$ Hypertriglyceridemia too leads to endothelial dysfunction that predisposes to the development of atherosclerotic depositions along vessel lumina. Visceral adiposity has been implicated in dysregulation of adiponectin levels, which too eventually causes vascular dysfunction. ${ }^{9}$

Thus, in multiple recent studies, it has been shown that platelet indices are higher in patients suffering from DM, impaired fasting glucose, and dyslipidemia, as compared with normal, healthy individuals. Thus, the aim of this study was to analyze the platelet indices in MetS patients.

\section{Objectives}

- To assess various platelet indices in MetS patients including plateletcrit, MPV, and PDW.

- To correlate platelet indices with other parameters of MetS, including waist circumference, BP, and lipid profile.

\section{Methods}

After obtaining due consent from the institutional ethics committee, a cross-sectional study was conducted in the Department of Medicine, and Central Clinical Laboratory, Acharya Vinoba Bhave Rural Hospital (AVBRH), Sawangi (Meghe), Wardha, for a period of 2 months from June 1, 2018 to July 31, 2018.

Study participants selected were 50 MetS patients aged between 18 and 60 years, attending the medicine OPD and/ or admitted to the medicine ward/intensive care unit (ICU). A control group of 50 healthy, non-MetS participants was also chosen. Participants were selected on the basis of simple random sampling to eliminate any bias.

\section{Diagnostic Criteria}

Diagnostic criteria used to classify MetS patients included the American College of Cardiology and American Health Association's National Cholesterol Education Program: modified Adult Treatment Plan III (NCEP-ATP III) criteria, ${ }^{10}$ which includes the following, and requires three or more positive findings to confirm a diagnosis:

- Abdominal obesity: Waist circumference $>80 \mathrm{~cm}$ in women and $>90 \mathrm{~cm}$ in men

- Body mass index (BMI): $>30 \mathrm{~kg} / \mathrm{m}^{2}$, and/or waist/hip ratio $>0.09$ in men and $>0.85$ in women

- Raised triglyceride levels: $\geq 150 \mathrm{mg} / \mathrm{dL}$

- Reduced HDL levels: $<40 \mathrm{mg} / \mathrm{dL}$ in men and $<50 \mathrm{mg} / \mathrm{dL}$ in women

- Raised BP: $\geq 130 \mathrm{~mm}$ Hg systolic or $\geq 85 \mathrm{~mm} \mathrm{Hg}$ diastolic

- Raised fasting glucose levels: $110-125 \mathrm{mg} / \mathrm{dL}$, type $2 \mathrm{DM}$ or impaired glucose tolerance and/or insulin resistance, denoted by hyperinsulinemia relative to glucose levels

Exclusion criteria considered were

- Patients suffering from coronary artery disease, stroke, or DM

- Patients having blood dyscrasias such as sickle cell trait or thalassemia

- Patients on treatment including glucocorticoids, anticoagulants, or antiplatelet drugs

Written consent of participants was then obtained, which explained in detail about the purpose, methodology, and implications of this study. Next, a proforma detailing relevant clinical history was taken.

\section{Measurement of Blood Pressure}

According to American Heart Association (AHA) guidelines, high BP is defined as readings of $\geq 130 \mathrm{~mm} \mathrm{Hg}$ for systolic blood pressure (SBP) measurement, or readings of $\geq 80$ for the diastolic blood pressure (DBP) measurement. BP of each patient was thus taken based on AHA-recommended protocol. ${ }^{11}$

\section{Measurement of Waist Circumference}

Waist circumference was measured at the midpoint between the lower margin of least palpable rib and the top of the iliac crest, using a stretch resistant tape that provided a constant 100 -g tension. The tape was fitted to be snug around the body, but not pulled so tight that it was constricting. The patient was made to stand with arms at the sides, feet positioned close together, and weight evenly distributed across the feet. Waist circumference was measured at the end of a normal expiration, when the lungs are at their functional residual capacity. The patient was advised to relax and take a few deep, natural breaths before the actual measurement was made, to minimize the pull of abdominal contents during the waist measurement. 


\section{Collection of Blood Sample}

Skin over the median cubital vein was first disinfected by applying surgical spirit over the cubital fossa, along with the application of a tourniquet proximal to the fossa. Then using a sterile standard venipuncture needle, blood was collected in a potassium ethylene diamine tetra acetate bulb for sampling and complete blood count, and was analyzed within 15 minutes of collection.

\section{Calculation of Mean Platelet Volume}

The blood collected was placed in a siliconed, graduated centrifuge tube containing $0.3 \mathrm{~mL}$ of $45 \%$ disodium ethylene diamine tetraacetic acid and mixed. Then the sample was spun at $900 \mathrm{rpm}$ (revolution per minute) for 15 minutes and supernatant plasma separated $20 \mathrm{~mm}$ of platelet-rich plasma obtained was then diluted in $100 \mathrm{~mL}$ of $0.85 \%$ saline giving a dilution ratio of $1 / 5,000$.

\section{Electronic Counting of Platelet Indices}

Based on the principle of detection of electrolyte displacement, platelet indices were then obtained from the $1 / 5,000$ dilution using a coulter counter (Beckman coulters $\mathrm{Z}$ series: $\mathrm{Z}$ counter manufactured by Beckman Coulter, Germany), with a 0.50 orifice, aperture current setting 7 , and threshold 25 .

\section{Calculation of Triglycerides}

Quantitative in vitro determination of triglycerides in serum or plasma was done by colorimetric method. The triglycerides were determined after enzymatic hydrolysis with lipases. The indicator used was a quinone imine formed from hydrogen peroxide, 4-aminophenazone, and 4-chlorophenol under the influence of peroxidase.

\section{Statistical Analysis}

Statistical analyses were carried out using inferential statistics, including chi-square test and Student's $t$-test. Software used for the analysis was SPSS version 22.0 (IBM Corporation, Armonk, New York, United States) and GraphPad Prism version 6.0 (Informer Technologies, Inc. Los Angeles, United States), with $p<0.05$ being considered as significant.

\section{Results}

This study assessed morphologic, hematologic, and biochemical parameters of 100 candidates who were chosen by simple random sampling. Category I comprised 50 participants who were diagnosed with MetS, and category II comprised 50 participants who served as healthy counterparts of the control group. Criteria assessed to make the diagnosis of MetS were the NCEP-ATP III criteria, ${ }^{10}$ with diagnostic components previously mentioned.

On assessment of waist circumference of categories I and II patients, category I patients had a mean waist circumference of $92.65 \pm 6.97 \mathrm{~cm}$ whereas category II patients had a mean waist circumference of $84.72 \pm 5.26 \mathrm{~cm}$ (-Table 1). On assessment by Student's unpaired $t$-test, a positive, significant correlation of waist circumference and MetS status of patients was obtained $(p=0.036)(-$ Table $\mathbf{1})$.

BP was the parameter assessed next. Mean SBP of category I patients was found to be $136.96 \pm 14.32 \mathrm{~mm} \mathrm{Hg}$, whereas mean SBP of category II patients was found to be $128.12 \pm 8.76 \mathrm{~mm} \mathrm{Hg}$. On applying Student's unpaired $t$-test, there was a positive, significant correlation found in relation to their MetS status $(p=0.022)$ ( $($ Table 2 ). On comparison of mean DBP, mean DBP of category I patients was found to be $89.20 \pm 07.32 \mathrm{~mm} \mathrm{Hg}$, whereas mean DBP of category II patients was found to be $85.92 \pm 05.28 \mathrm{~mm} \mathrm{Hg}$. On assessment by Student's unpaired $t$-test, there was no correlation found between DBP of patients and their MetS status.

Lipid profile of patients was then assessed. When serum triglyceride levels of categories I and II patients were compared, mean serum triglyceride levels of category I patients were found to be $178.53 \pm 68.91 \mathrm{mg} / \mathrm{dL}$, whereas mean serum triglyceride levels of category II patients were found to be $140.57 \pm 49.82 \mathrm{mg} / \mathrm{dL}$. On applying $t$-test, a positive, significant correlation was found in relation to their MetS status $(p=0.035)$ (-Table 3$)$. When mean serum HDL levels were compared among the two categories, mean serum HDL levels of category I patients were found to be $28.95 \pm 7.40 \mathrm{mg} / \mathrm{dL}$, whereas mean serum HDL levels of category II patients were found to be $34.16 \pm 8.06 \mathrm{mg} / \mathrm{dL}$. On assessment by Student's unpaired $t$-test, no correlation was found between mean serum HDL levels of patients and their MetS status.

Fasting blood glucose levels of patients was the subsequent parameter taken into consideration. When fasting blood glucose of the two categories was compared, mean fasting blood glucose levels of category I patients were found to be 143.34 $\pm 72.86 \mathrm{mg} / \mathrm{dL}$, whereas mean fasting blood glucose levels of category II patients were found to be $123.39 \pm 40.04 \mathrm{mg} / \mathrm{dL}$. On assessment by Student's unpaired $t$-test, there was no

Table 1 Correlation of metabolic syndrome status and waist circumference of study population

\begin{tabular}{|l|l|l|l|l|l|l|}
\hline Category & $N$ & Mean & Standard deviation & Standard error mean & $t$-Value & $p$-Value \\
\hline I II & 50 & 92.65 & 6.97 & 1.50 & 2.12 & $0.036, \mathrm{~S}$ \\
\end{tabular}

Table 2 Correlation of metabolic syndrome status and systolic blood pressure of study population

\begin{tabular}{|l|l|l|l|l|l|l|}
\hline Category & $N$ & Mean & Standard deviation & Standard error mean & $t$-Value & $p$-Value \\
\hline I & 50 & 136.96 & 14.32 & 1.86 & 2.33 & $0.022, S$ \\
\end{tabular}


Table 3 Correlation of metabolic syndrome status and serum triglyceride levels of study population

\begin{tabular}{|l|l|l|l|l|l|l|}
\hline Category & $N$ & Mean & Standard deviation & Standard error mean & $t$-Value & $p$-Value \\
\hline I & 50 & 178.53 & 68.91 & 9.69 & 2.09 & 0.035, S \\
\hline II & 50 & 140.57 & 49.82 & 5.97 & & \\
\hline
\end{tabular}

correlation found between fasting blood glucose of patients and their MetS status.

Platelet indices of patients, comprising plateletcrit, MPV, and PDW, were then taken into consideration. The first parameter taken into consideration was the patient's plateletcrit. When mean plateletcrit of the two categories was compared, mean plateletcrit levels of category I patients were found to be $0.25 \pm 0.08 \%$, whereas mean plateletcrit levels of category II patients were found to be $0.21 \pm 0.02 \%$. When these values were assessed using Student's unpaired $t$-test, a positive, significant correlation was found between mean plateletcrit of patients in correlation to their MetS status $(p=0.019, \mathrm{~S})$ (-Table 4$)$. When MPV of the two categories was compared, MPV of category I patients was found to be $10.07 \pm 1.05 \mathrm{fL}$, whereas MPV of category II patients was found to be $10.23 \pm 0.66 \mathrm{fL}$. On assessment by Student's unpaired $t$-test, no correlation was found between MPV of patients and their MetS status. The last platelet parameter to be assessed was PDW. When mean PDW of the two categories was compared, mean PDW of category I patients was found to be $15.37 \pm 2.66 \%$, whereas mean PDW of category II patients was found to be $14.82 \pm 2.15 \%$. On assessment by Student's unpaired $t$-test, no correlation was found between mean PDW of patients and their MetS status.

\section{Discussion}

Out of the 100 study patients chosen by simple random sampling, category I consisted of 50 patients who were MetS patients and category II comprised 50 healthy control group participants. Thirty-four (68\%) of category I patients were males, whereas 16 (32\%) were females. Twenty-eight (56\%) of category II patients were males, whereas 22 (44\%) were females. On assessment by Student's unpaired $t$-test, no statistical significance was found in correlation of sex of patients to MetS status $(p<0.05)$. When patients' age was assessed, $5(10 \%)$ category I patients were from the age group of 18 to 30 years, 14 (28\%) belonged to the age group of 30 to 40 years, a maximum of 19 (38\%) were between 40 and 50 years, whereas 12 (24\%) lay in the age group of 50 to 60 years. Out of category II patients, 11 patients (22\%) belonged to the age group of 18 to 30 years, 18 (36\%) belonged to the age group of 30 to 40 years, 14 (28\%) were between 40 and 50 years of age, whereas 7 (14\%) lay in the age group of 50 to 60 years. On assessment by Student's unpaired $t$-test, no statistical significance was found in correlation of patients' age to their MetS status $(p<0.05)$.

The findings we obtained in this study have been similar to those obtained by various other authors regarding work on both, MetS, and platelet indices in national and international literature. We obtained a significant, positive correlation of the MetS status of patients to their waist circumference, SBP, serum triglyceride levels, and plateletcrit, as confirmed using independent Student's unpaired $t$-tests.

Fan et al demonstrated that the levels of endothelial microparticles, PMPs (platelet-derived microparticles), and microparticle-carried protein disulfide isomerase (PDI) activity are found to be significantly increased in MetS patients. ${ }^{12}$ The role of platelet-activation pathways has also been widely studied in causation of complications in MetS. ${ }^{13,14}$ Studies have shown that platelet aggregation is controlled by the activity of PDI. This relationship between PDI and platelet hyperreactivity in MetS and its role in the causation of insulin resistance and nitric oxide dysfunction were studied by Gaspar et al..$^{15}$

Further pathophysiology of platelet dysfunction and other biochemical abnormalities in MetS patients was studied by Santilli et al, who documented sex-based differences in cardiovascular risk factors and predisposition to DM. ${ }^{16}$ This sex-based female predisposition was noted by authors Zhou et al as well, who have shown that increasing plateletcrit acts as a predisposing factor to development of cardiovascular complications in MetS patients, and also noted an increased risk of developing MetS among patients with increased leucocyte and erythrocyte counts. ${ }^{17,18}$ Furman et al noted that elevated platelet indices correlated statistically with waist circumferences of patients and acted as a determinant of abdominal obesity, a major factor implicated in the development of MetS, with similar results shown by authors Barzin et al as well. ${ }^{19,20}$

Other platelet indices studied include platelet-tolymphocyte ratio, which was found to be elevated in MetS patients, as well as shown be a marker for cardiovascular dysfunction in MetS patients by Akboga et al. ${ }^{21,22} \mathrm{~A}$ large multicenter study based in South Korea, spanning 2 years from 2010 to 2012 and involving 2,228 patients by Lim et al, showed that elevated platelet indices were a risk stratification marker for development of MetS not only in adults but in the pediatric and adolescent population as well. ${ }^{23}$ Similar results on proinflammatory biomarkers in MetS patients in the adolescent age group were documented by Can et al. ${ }^{24}$

Table 4 Correlation of metabolic syndrome status and plateletcrit of study population

\begin{tabular}{|l|l|l|l|l|l|l|}
\hline Category & $N$ & Mean & Standard deviation & Standard error mean & $t$-Value & $p$-Value \\
\cline { 1 - 5 } I II & 50 & 0.25 & 0.08 & 0.014 & 2.49 & $0.019, \mathrm{~S}$ \\
\end{tabular}


On comparison with controls, Zaccardi et al found that MPV was significantly higher in patients with type 2 DM but not in MetS patients, findings similar to what were obtained in our study as well. ${ }^{25,26}$

\section{Conclusion}

Thus, this study demonstrated that MetS is a proinflammatory and prothrombotic state, characterized by alteration of platelet indices. Plateletcrit was shown to be a significant biomarker along with other parameters, including waist circumference, SBP, and serum triglyceride levels. Early detection using these markers can lead to an overall decline in morbidity and mortality due to MetS.

\section{Funding}

None.

\section{Conflict of Interest}

None declared.

\section{References}

1 Pickering TG, Hall JE, Appel LJ, et al. Recommendations for blood pressure measurement in humans and experimental animals: part 1: blood pressure measurement in humans: a statement for professionals from the Subcommittee of Professional and Public Education of the American Heart Association Council on High Blood Pressure Research. Circulation 2005;111(5):697-716

2 Ramachandran A, Snehalatha C, Satyavani K, Sivasankari S, Vijay V. Metabolic syndrome in urban Asian Indian adults-a population study using modified ATP III criteria. Diabetes Res Clin Pract 2003;60(3):199-204

3 Misra A, Misra R, Wijesuriya M, Banerjee D. The metabolic syndrome in South Asians: continuing escalation \& possible solutions. Indian J Med Res 2007;125(3):345-354

4 Misra A, Khurana L. Obesity and the metabolic syndrome in developing countries. J Clin Endocrinol Metab 2008;93 (11, Suppl 1):S9-S30

5 Kanjilal S, Shanker J, Rao VS, et al. Prevalence and component analysis of metabolic syndrome: an Indian atherosclerosis research study perspective. Vasc Health Risk Manag 2008;4(1):189-197

6 Das M, Pal S, Ghosh A. Association of metabolic syndrome with obesity measures, metabolic profiles, and intake of dietary fatty acids in people of Asian Indian origin. J Cardiovasc Dis Res 2010;1(3):130-135

7 Pemminati S, Prabha Adhikari MR, Pathak R, Pai MR. Prevalence of metabolic syndrome using IDF 2005 guidelines in a semi-urban south Indian population of Mangalore. J Assoc Physicians India 2010;58:674-677

8 Ford ES. The metabolic syndrome and mortality from cardiovascular disease and all-causes: findings from the National Health and Nutrition Examination Survey II Mortality Study. Atherosclerosis 2004;173(2):309-314

9 Ryo M, Nakamura T, Kihara S, et al. Adiponectin as a biomarker of the metabolic syndrome. Circ J 2004;68(11):975-981

10 Grundy SM, Cleeman JI, Daniels SR, et al; American Heart Association; National Heart, Lung, and Blood Institute. Diagnosis and management of the metabolic syndrome: an American Heart Association/National Heart, Lung, and Blood Institute scientific statement. Circulation 2005;112(17):2735-2752

11 Perloff D, Grim C, Flack J, et al. Human blood pressure determination by sphygmomanometry. Circulation 1993;88(5 Pt 1): 2460-2470

12 Fan GQ Qin RR, Li YH, et al. Endothelial cells microparticle-associated protein disulfide isomerase promotes platelet activation in metabolic syndrome. Oncotarget 2016;7(50):83231-83240

13 van Rooy MJ, Pretorius E. Metabolic syndrome, platelet activation and the development of transient ischemic attack or thromboembolic stroke. Thromb Res 2015;135(3):434-442

14 Koren-Morag N, Goldbourt U, Tanne D. Relation between the metabolic syndrome and ischemic stroke or transient ischemic attack: a prospective cohort study in patients with atherosclerotic cardiovascular disease. Stroke 2005;36(7):1366-1371

15 Gaspar RS, Trostchansky A, Paes AM. Potential role of protein disulfide isomerase in metabolic syndrome-derived platelet hyperactivity. Oxid Med Cell Longev 2016;2016:2423547

16 Santilli F, D’Ardes D, Guagnano MT, Davi G. Metabolic syndrome: sex-related cardiovascular risk and therapeutic approach. Curr Med Chem 2017;24(24):2602-2627

17 Zhou P, Meng Z, Liu M, et al. The associations between leukocyte, erythrocyte or platelet, and metabolic syndrome in different genders of Chinese. Medicine (Baltimore) 2016;95(44):e5189

18 Zhao F, Yan Z, Meng Z, et al. Relationship between mean platelet volume and metabolic syndrome in Chinese patients. Sci Rep 2018;8(1):14574

19 Furman-Niedziejko A, Rostoff P, Rychlak R, et al. Relationship between abdominal obesity, platelet blood count and mean platelet volume in patients with metabolic syndrome. Folia Med Cracov 2014;54(2):55-64

20 Barzin M, Asghari G, Hosseinpanah F, Mirmiran P, Azizi F. The association of anthropometric indices in adolescence with the occurrence of the metabolic syndrome in early adulthood: Tehran Lipid and Glucose Study (TLGS). Pediatr Obes 2013;8(3):170-177

21 Akboga MK, Canpolat U, Yuksel M, et al. Platelet to lymphocyte ratio as a novel indicator of inflammation is correlated with the severity of metabolic syndrome: a single center large-scale study. Platelets 2016;27(2):178-183

22 Akboga MK, Canpolat U, Yayla C, et al. Association of platelet to lymphocyte ratio with inflammation and severity of coronary atherosclerosis in patients with stable coronary artery disease. Angiology 2016;67(1):89-95

23 Lim HJ, Seo MS, Shim JY, Kim KE, Shin YH, Lee YJ. The association between platelet count and metabolic syndrome in children and adolescents. Platelets 2015;26(8):758-763

24 Can U, Buyukinan M, Guzelant A, Ugur A, Karaibrahimoglu A, Yabanciun $S$. Investigation of the inflammatory biomarkers of metabolic syndrome in adolescents. J Pediatr Endocrinol Metab 2016;29(11):1277-1283

25 Zaccardi F, Rocca B, Pitocco D, Tanese L, Rizzi A, Ghirlanda G. Platelet mean volume, distribution width, and count in type 2 diabetes, impaired fasting glucose, and metabolic syndrome: a meta-analysis. Diabetes Metab Res Rev 2015;31(4):402-410

26 Coban E, Bostan F, Ozdogan M. The mean platelet volume in subjects with impaired fasting glucose. Platelets 2006;17(1):67-69 